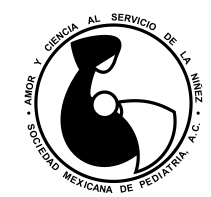

\title{
Los alcances de la investigación clínica
}

\author{
Jessi Nayelli Zurita-Cruz ${ }^{1, *}$ \\ ${ }^{1}$ Unidad de Investigación Médica en Nutrición, UMAE Hospital de Pediatría \\ Centro Médico Nacional Siglo XXI, Instituto Mexicano del Seguro Social, Ciudad de México.
}

La investigación clínica es la disciplina que estudia tanto pacientes de manera integral como sus datos personales, imágenes o muestras de tejido, para entender la salud y la enfermedad. De acuerdo con el objetivo de la investigación clínica, se puede clasificar en diferentes tipos como: tratamiento, prevención, diagnóstico, etiología y epidemiológicos, pero también puede incluir estudios explicativos para conocer la fisiopatología.

La participación de pacientes en la investigación clínica no excluye el empleo de técnicas de investigación básica; de hecho, cada vez es más frecuente que ambas disciplinas combinen sus conocimientos, y se nombra como investigación traslacional. Un ejemplo de la investigación traslacional es la investigación clínica con enfoque genético, como los microARNs (miRNAs) que son una clase de pequeñas moléculas de ARN endógenas que se han visto relacionadas con una menor supervivencia en pacientes con enfermedades oncológicas.

Las investigaciones clínicas ayudan a encontrar nuevas y mejores formas de detección, diagnóstico, tratamiento, pronóstico y prevención de una enfermedad. Uno de los diseños prototipo en la investigación clínica lo constituyen los ensayos clínicos, en los que se prueban tratamientos (que pueden ser nuevos) para una enfermedad. Mientras que, en los estudios de pronóstico, uno de los objetivos se centra en determinar la evolución natural de una enfermedad mediante la vigilancia de una cohorte a lo largo del tiempo, a fin de recabar información para entender cómo evoluciona.

\footnotetext{
* Correspondencia: JNZC, zuritajn@hotmail.com

Conflicto de intereses: La autora declara que no tiene.

Citar como: Zurita-Cruz JN. Los alcances de la investigación clínica. Rev Mex Pediatr. 2019; 86(5):173-174.

[The scope of clinical research]
}

La participación de seres humanos en la investigación requiere una estrecha atención a todos los aspectos éticos y de seguridad. En este aspecto debemos enfatizar los requisitos indispensables para una investigación clínica bien hecha desde un punto de vista ético, en el que se incluye el valor científico y social, selección equitativa de los sujetos, una adecuada evaluación del riesgo/beneficio, contar con consentimiento y asentimiento informado, a fin de que en todo momento se respete la autonomía de los individuos reclutados en el estudio.

La investigación clínica es el último vínculo de la secuencia en la investigación biomédica. Cualquier conocimiento que se genera en la investigación básica se debe comprobar en la investigación clínica para que se obtenga un impacto en la práctica médica diaria. Retomando los miRNAs, los cuales fueron identificados en investigaciones básicas, al analizarse en humanos se detectó que se encuentran circulando en la sangre, lo que representa una oportunidad para usarlos como biomarcadores. Así, tras realizar estudios con miRNAs se ha comprobado la utilidad de estos biomarcadores para llevar a cabo diagnósticos más tempranos de cáncer de mama metastásico, así como su monitorización. Por lo tanto, la investigación clínica es de suma importancia para comprobar lo descrito en la investigación básica.

Es fundamental el conocimiento de los aspectos clínicos de la enfermedad que se quiere investigar, a fin de plantear una adecuada pregunta de investigación y analizar los resultados en el contexto de dicha enfermedad. De esta forma, en la investigación clínica lo primero es plantear la pregunta y posteriormente identificar los mejores métodos para contestarla; sin embargo, es frecuente caer en el error de contestar la pregunta utilizando los métodos o técnicas disponibles en un laboratorio u hospital determinado y no con base en la necesidad de generar conocimiento nuevo. Este tipo de error puede condicionar que los resultados 
obtenidos de la investigación sean poco relevantes. Ante esto, es importante enfatizar que el proceso de investigación clínica debe iniciar por la formulación de la pregunta y posteriormente identificar la mejor forma de contestarla que puede ser con innovación. Por ejemplo, para detectar deterioro de la función renal en pacientes con enfermedad renal crónica estadio I a IV, se ha considerado de utilidad el uso de microalbuminuria o el índice proteína/creatinina; sin embargo, son marcadores de progresión renal que ya han demostrado su utilidad en múltiples estudios, por lo que valdría la pena utilizar algún marcador más reciente como la podocalixina, en lugar de los que ya han demostrado que sirven.

En la investigación clínica se deben tener conocimientos básicos sobre la metodología de la investigación con la finalidad de identificar el mejor diseño de investigación para poder contestar la pregunta de investigación, definir claramente los resultados y la relevancia clínica, además de establecer un tamaño de muestra adecuado y tener la capacidad para realizar análisis estadísticos apropiados. Dentro de este contexto, es importante saber diferenciar entre la significancia estadística y la significancia clínica. Si bien, un resultado experimental puede ser muy consistente y reproducible (lo que daría una $\mathrm{p}<0.05$ ), pero de escasa relevancia clínica. Por ejemplo, en los resultados de un tratamiento farmacológico innovador para bajar de peso en sujetos con obesidad se demuestran una pérdida de peso de 500 gramos posterior a seis meses de manejo con significancia estadística en comparación con otro tratamiento; no obstante, la disminución de 500 gramos en este tipo de individuos no tiene relevancia clínica para mejorar las comorbilidades cardiometabólicas. Por lo que uno de los grandes retos de la investigación clínica es obtener resultados con relevancia clínica y no sólo con significancia estadística.

Por último, se debe reflexionar que la gran mayoría de los estudios de investigación clínica no están diseñados para beneficiar directamente a los participantes; sin embargo, realizarlos ayuda en la búsqueda de alternativas para mejorar la salud de la población.

\section{BIBLIOGRAFÍA RECOMENDADA}

1. Lenfant $\mathrm{C}$. Shattuck lecture--clinical research to clinical practice-lost in translation? N Engl J Med. 2003; 349: 868-874.

2. Miranda-Novales MG, Villasís-Keever MÁ. El protocolo de investigación. Parte I. Rev Alerg Mex. 2015; 62 (4): 312-317.

3. Joshi P, Katsushima K, Zhou R, Meoded A, Stapleton S, Jallo G et al. The therapeutic and diagnostic potential of regulatory noncoding RNAs in medulloblastoma. Neurooncol Adv. 2019; 1(1): vdz023.

4. Mulholland EJ, Green WP, Buckley NE, McCarthy HO. Exploring the potential of MicroRNA Let-7c as a therapeutic for prostate cancer. Mol Ther Nucleic Acids. 2019; 18: 927-937.

5. McGuire A, Brown JA, Kerin MJ. Metastatic breast cancer: the potential of miRNA for diagnosis and treatment monitoring. Cancer Metastasis Rev. 2015; 34(1): 145-155.

6. Marino AL, Evangelista AF, Vieira RA, Macedo T, Kerr LM, Abrahão-Machado LF et al. MicroRNA expression as risk biomarker of breast cancer metastasis: a pilot retrospective casecohort study. BMC Cancer. 2014; 14: 739.

7. Nielsen JS, McNagny KM. The role of podocalyxin in health and disease. J Am Soc Nephrol. 2009; 20(8): 1669-1676.

8. Erkan E. Proteinuria and progression of glomerular diseases. Pediatric Nephrology. 2013; 28(7): 1049-1058.

9. Varmus $\mathrm{H}$. Shattuck lecture- biomedical research enters the steady state. N Engl J Med. 1995; 333: 811-815.

10. Moses H3rd, Martin JB. Biomedical research and health advances. N Engl J Med. 2011; 364(6): 567-571. 